\title{
Metaplastic Breast Cancer: To Radiate or Not to Radiate?
}

\author{
Warren H. Tseng, MD and Steve R. Martinez, MD, MAS \\ Division of Surgical Oncology, Department of Surgery, University of California Davis, Sacramento, CA
}

\begin{abstract}
Background. The role of radiation therapy (RT) is unclear for metaplastic breast cancer (MBC). We hypothesized that RT would improve overall survival (OS) and disease-specific survival (DSS).

Materials and Methods. We used the Surveillance, Epidemiology, and End Results (SEER) database to identify MBC patients diagnosed from1988 to 2006. Univariate analyses of patient, tumor, and treatment-specific factors on OS and DSS were performed using the Kaplan-Meier method and differences among survival curves assessed via $\log$ rank. Variables assessed included patient age, race/ ethnicity, histologic subtype, tumor grade, $\mathrm{T}$ stage, $\mathrm{N}$ stage, M stage, hormone receptor status, surgery type, and use of RT. Cox proportional hazards models used all univariate covariates. Risks of mortality were reported as hazard ratios (HR) with 95\% confidence intervals (95\% CI); significance was set at $P \leq 0.05$.
\end{abstract}

Results. Among 1501 patients, RT was given to 580 (38.6\%). Ten-year OS and DSS were 53.2, and 68.3\%, respectively. In the overall analysis, RT provided an OS (HR 0.64; 95\% CI, 0.51-0.82; $P<0.001$ ) and DSS (HR 0.74 ; CI, $0.56-0.96 ; P<0.03$ ) benefit. When patients were stratified according to type of surgery, RT provided an OS but not a DSS benefit to lumpectomy (HR 0.51; CI, 0.32$0.79, P<0.01)$ and mastectomy patients (HR 0.67; CI, $0.49-0.90 ; P<0.01)$.

Presented in part at the Society of Surgical Oncology 63rd Annual Cancer Symposium, St. Louis, MO March 4-7, 2010.

(C) The Author(s) 2010. This article is published with open access at Springerlink.com

First Received: 27 February 2010;

Published Online: 29 June 2010

S. R. Martinez, MD, MAS

e-mail: steve.martinez@ucdmc.ucdavis.edu
Conclusions. Our findings support the use of RT for patients with MBC following lumpectomy or mastectomy. These retrospective findings should be confirmed in a prospective clinical trial.

Metaplastic breast cancer (MBC) is characterized by the histologic presence of 2 or more cellular types commonly representing a mixture of epithelial and mesenchymal components. ${ }^{1-6} \mathrm{MBC}$ represents $0.25-1 \%$ of breast cancers diagnosed annually. ${ }^{1,7}$ The prognosis of and optimal treatment for MBC are largely unknown. Review of more than 800 MBC patients in the National Cancer Database from 2001 to 2003 indicates that, compared with patients with invasive ductal carcinoma (IDC), those with MBC have larger, higher-grade and more hormone receptor negative tumors with less involvement of regional lymph nodes. ${ }^{8}$ Overall, more than $70 \%$ of patients with MBC present with American Joint Committee on Cancer (AJCC) stage II or greater disease, compared with approximately $50 \%$ of patients with IDC. ${ }^{8}$

The World Health Organization (WHO) recognized MBC as a unique pathologic entity in 2000. Since then, the incidence of MBC has increased, presumably because of increased recognition by pathologists. ${ }^{8,9}$ Management of MBC has largely paralleled that of IDC. Compared with patients with IDC, however, those with MBC have worse outcomes with 5 -year survival rates ranging from 49 to $68 \%$. $^{1,10,11}$

Information regarding the role of adjuvant radiation therapy (RT) for the treatment of MBC is sparse. ${ }^{12,13}$ The Breast Disease Site Team reported that only $42.5 \%$ of their MBC patients received RT compared with $52.0 \%$ of IDC patients, but outcome data were not reported. ${ }^{8}$ Given the aggressive features of MBC relative to IDC and the dearth of information concerning the role of RT, we sought to use a large, population-based cancer registry to analyze the effects of RT on overall (OS) and disease-specific survival (DSS) in patients with MBC. 


\section{METHODS}

The Surveillance, Epidemiology, and End Results (SEER) database of the National Cancer Institute was used to identify all patients with MBC diagnosed from 1988 to 2006. SEER collects cancer incidence and survival data from 17 population-based cancer registries representing $26 \%$ of the U.S. population.

All cases of primary, histologically confirmed, MBC were eligible. We used SEER ICD-0-3 codes 8560, 8562, $8570-8572,8575$, and 8980-8982. Patients identified by death certificate or autopsy, and those with missing cause of death were excluded. The final sample included 1501 patients.

Survival time was calculated as the number of completed months between the date of diagnosis and whichever occurred first: date of death, date last known to be alive, or December 31, 2006. The endpoints for the present study were OS and DSS. For calculations of OS and DSS, patients who were lost to follow-up or survived beyond December 31, 2006 were coded as censored observations. Similarly, for calculations of DSS, patients who died from non-breastrelated causes were coded as censored observations.

We compared univariate rates of OS and DSS according to use of RT using the Kaplan-Meier method. We further stratified patients by surgery type: lumpectomy versus mastectomy. Additional univariate survival comparisons were made for the following patient, tumor, and treatmentspecific factors: age at diagnosis, race/ethnicity, histologic subtype, tumor grade, $\mathrm{T}$ stage (tumor size in $\mathrm{cm}$ ), $\mathrm{N}$ stage (number of positive lymph nodes), $\mathrm{M}$ stage (presence of distant metastasis), hormone receptor status, type of primary surgery (lumpectomy or mastectomy), and use of RT (radiation, no radiation, unknown). Differences between or among survival curves were assessed via the log-rank test.

Cox proportional hazards models assessed the effect of RT on OS and DSS. All univariate factors were included in the multivariate model. For purposes of further evaluation of the effect of RT, we stratified patients according to whether they received lumpectomy or mastectomy as primary surgical treatment and performed subgroup multivariate analyses. Unknown or missing data were presented and analyzed concomitantly.

To examine whether the breast cancers described prior to recognition of $\mathrm{MBC}$ as a distinct pathologic entity by the WHO in 2000 were similar to those diagnosed after WHO recognition, patients were divided into 2 eras. Those diagnosed with MBC in 2000 or earlier were placed in era I, while those diagnosed from 2001 to 2006 were placed in era II. Univariate and multivariate analyses compared patients from the 2 treatment eras.

Risks of overall and disease-specific mortality were reported as hazard ratios (HR) with $95 \%$ confidence intervals $(95 \% \mathrm{CI})$; significance was set at $P \leq 0.05$. All statistical analyses were two-tailed and performed using STATA version 10 (StataCorp, College Station, TX) or SPSS (SPSS, Inc., Chicago, IL).

This study was exempt from institutional review board (IRB) review.

\section{RESULTS}

Entry criteria were met by 1501 patients. Characteristics of the study population are presented in Table 1. Briefly, the majority of patients were white $(73.0 \%)$ women (99.9\%). Multiple histologic subtypes were represented. Prevalent histologies included: metaplastic not otherwise specified (57.3\%), adenosquamous carcinoma (18.1\%), and carcinosarcoma (11.7\%). Only $3.9 \%$ of patients had an unknown $\mathrm{T}$ stage, while $68.7 \%$ had tumors greater than $2 \mathrm{~cm}$ in diameter (T2, T3, or T4 tumors). Nodal status was unknown for $15.3 \% ; 22.5 \%$ were node positive. A majority of patients presented without evidence of distant metastases $(92.5 \%)$. MBC tumors were most commonly of higher grade $(67.8 \%$ ), although $20.2 \%$ had unknown tumor grade. Estrogen receptor and progesterone receptor status was negative in 69.2 and $69.9 \%$, respectively. Estrogen receptor status was unknown for $17.8 \%$, and progesterone receptor status was unknown for $18.5 \%$. Patients were most commonly treated with mastectomy $(55.5 \%) ; 41.0 \%$ of patients received lumpectomy while $2.9 \%$ did not undergo potentially curative surgery. Patients with T3 or T4 tumors were more likely to receive mastectomy $(83.4 \%)$ than were patients with smaller tumors (46.5\%). Similarly, $74.3 \%$ of node-positive patients received mastectomy. Radiation therapy was administered to $38.6 \%$ of patients; $23.4 \%$ of mastectomy patients and $61.8 \%$ of lumpectomy patients received RT.

Overall survival of the entire cohort was $63.3 \%$ at 5 years and $53.2 \%$ at 10 years. Disease-specific survival of the cohort was $71.8 \%$ at 5 years and $68.3 \%$ at 10 years. In patients receiving RT, OS was $73.2 \%$ at 5 years and $60.3 \%$ at 10 years versus 56.9 and $48.3 \%$ in patients not receiving RT (Fig. 1a). DSS for patients receiving RT was $75.9 \%$ at 5 years and $71.7 \%$ at 10 years compared with 69.0 and $66.1 \%$ in patients not receiving RT (Fig. 1b).

Patients receiving lumpectomy and RT demonstrated superior OS and DSS to similar patients not receiving RT ( $P<0.001$ and $P=0.003$, respectively) on univariate analysis. OS for patients receiving lumpectomy and RT was $65.5 \%$ at 10 years, while OS for those not receiving RT was $59.3 \%$ (Fig. 2a). DSS for patients receiving lumpectomy and RT was $79.4 \%$ at 10 years while DSS for those not receiving RT was $70.7 \%$ (Fig. 2b). Conversely, on univariate analysis, patients treated with mastectomy 
TABLE 1 Clinicopathologic characteristics; $n=1501$

\begin{tabular}{|c|c|}
\hline Variable & Percentage or mean \\
\hline Female & 99.9 \\
\hline Age & 62 \\
\hline \multicolumn{2}{|l|}{ Ethnicity/race } \\
\hline White & 73.0 \\
\hline Black & 14.3 \\
\hline Asian/Pacific Islander & 7.0 \\
\hline Hispanic & 5.2 \\
\hline Unknown & 0.5 \\
\hline \multicolumn{2}{|l|}{ Histologic subtype } \\
\hline Metaplastic NOS & 57.3 \\
\hline Adenosquamous & 18.1 \\
\hline Carcinosarcoma & 11.7 \\
\hline Adenospindle/cartilaginous/osseus & 9.3 \\
\hline Epithelial/myoepithelial & 3.6 \\
\hline \multicolumn{2}{|l|}{ T stage } \\
\hline $\mathrm{T} 1$ & 27.4 \\
\hline $\mathrm{T} 2$ & 44.6 \\
\hline $\mathrm{T} 3$ & 12.5 \\
\hline $\mathrm{T} 4$ & 11.6 \\
\hline Unknown & 3.9 \\
\hline \multicolumn{2}{|l|}{$\mathrm{N}$ stage } \\
\hline No & 62.2 \\
\hline N1 & 15.1 \\
\hline $\mathrm{N} 2$ & 5.2 \\
\hline N3 & 2.2 \\
\hline Unknown & 15.3 \\
\hline \multicolumn{2}{|l|}{ M stage } \\
\hline M0 & 92.5 \\
\hline M1 & 6.1 \\
\hline Unknown & 1.3 \\
\hline \multicolumn{2}{|l|}{ Tumor grade } \\
\hline $\mathrm{I} / \mathrm{II}$ & 12.1 \\
\hline III/IV & 67.8 \\
\hline Unknown & 20.2 \\
\hline \multicolumn{2}{|l|}{ Hormonal receptor status } \\
\hline ER positive & 12.7 \\
\hline ER negative & 69.2 \\
\hline ER unknown & 17.8 \\
\hline PR positive & 11.5 \\
\hline PR negative & 69.9 \\
\hline PR unknown & 18.5 \\
\hline \multicolumn{2}{|l|}{ Surgery type } \\
\hline Lumpectomy & 41.0 \\
\hline Mastectomy & 55.5 \\
\hline None & 2.9 \\
\hline Unknown & 0.3 \\
\hline \multicolumn{2}{|l|}{ Radiation therapy } \\
\hline Yes & 38.6 \\
\hline No & 61.3 \\
\hline Unknown & 0.1 \\
\hline
\end{tabular}

and RT did not demonstrate OS or DSS benefits compared with their counterparts who did not receive RT $(P=0.49$ and $P=0.22$, respectively). The 10 -year OS rate for mastectomy patients receiving RT was 47.7 and $45.3 \%$ in patients not receiving RT (Fig. 3a). The 10-year DSS rate for mastectomy patients receiving RT was 55.0 and $65.3 \%$ in patients not receiving RT (Fig. 3b).

Factors influencing OS on univariate analysis included histologic subtype $(P=0.002)$, tumor grade $(P<0.001)$, T stage $(P<0.001)$, N stage $(P<0.001)$, M stage $(P<$ $0.001)$, surgery type $(P<0.001)$, and RT $(P<0.001)$. Univariate factors influencing DSS included race/ethnicity $(P=0.03)$, histologic subtype $(P=0.04)$, tumor grade $(P<0.001)$, T stage $(P<0.001)$, N stage $(P<0.001), \mathrm{M}$ stage $(P<0.001)$, surgery type $(P<0.001)$, and RT $(P<$ $0.01)$

Results of the entire cohort multivariate analysis for OS are presented in Table 2A. Factors increasing the risk of death due to any cause included increasing age (HR 1.02, 95\% CI 1.02-1.03; $P<0.01)$, carcinosarcoma histology (HR 1.52, 95\% CI 1.13-2.04; $P=0.005$ ), T3 (HR 2.82, 95\% CI 2.12-3.75; $P<0.01$ ) and T4 tumors (HR 1.95, 95\% CI 1.45-2.61; $P<0.01$ ), N1 status (HR 2.40, 95\% CI $1.83-3.15 ; P<0.01$ ), N2 status (HR 2.71, 95\% CI 1.834.02; $P<0.01$ ), N3 status (HR 1.92, 95\% CI 1.11-3.33; $P=0.02$ ), unknown nodal status (HR 2.32, 95\% CI 1.763.06; $P<0.01$ ), and distant metastases (HR 4.73, $95 \% \mathrm{CI}$ 3.51-6.39; $P<0.01)$. Factors decreasing the risk of death due to any cause included T1 tumors (HR 0.68, 95\% CI $0.50-0.93 ; P<0.01)$ and use of RT (HR $0.64,95 \% \mathrm{CI}$ $0.51-0.82 ; P<0.01)$.

Results of the entire cohort multivariate analysis for DSS are similarly presented in Table 2A. Factors increasing the risk of disease-related death included increasing age (HR 1.01, 95\% CI 1.00-1.02; $P<0.001$ ), carcinosarcoma histology (HR 1.63, 95\% CI 1.16-2.31, $P=0.005)$, T3 (HR 2.73, 95\% CI 1.94-3.86; $P<0.01$ ) and T4 tumors (HR 2.28, 95\% CI 1.62-3.21; $P<0.01$ ), N1 status (HR 2.71, 95\% CI 1.98-3.72; $P<0.01$ ), N2 status (HR 3.76, 95\% CI 2.45-5.77; $P<0.01$ ), N3 status (HR 2.40, 95\% CI 1.33-4.34; $P<0.01$ ), unknown nodal status (HR 2.24, 95\% CI 1.58-3.20; $P<0.01$ ), and distant metastases (HR 5.32, 95\% CI 3.80-7.45; $P<0.01)$. Factors decreasing the risk of disease-related death included T1 tumors (HR 0.59, 95\% CI $0.39-0.88 ; P<0.01)$, lower grade (I/II) tumors (HR $0.51,95 \%$ CI $0.29-0.89 ; P=0.02$ ), and use of RT (HR $0.74,95 \%$ CI $0.56-0.96 ; P<0.03$ ).

Multivariate analysis was repeated after stratifying patients according to whether they received lumpectomy (Table 2B) or mastectomy (Table 2C). The influence of RT on OS and DSS in lumpectomy and mastectomy groups is summarized in Table 3. Within the lumpectomy cohort, RT was associated with an OS benefit (HR 0.51, 95\% CI $0.32-$ 
FIG. 1 a Overall survival-entire cohort. b Breast-specific survivalentire cohort

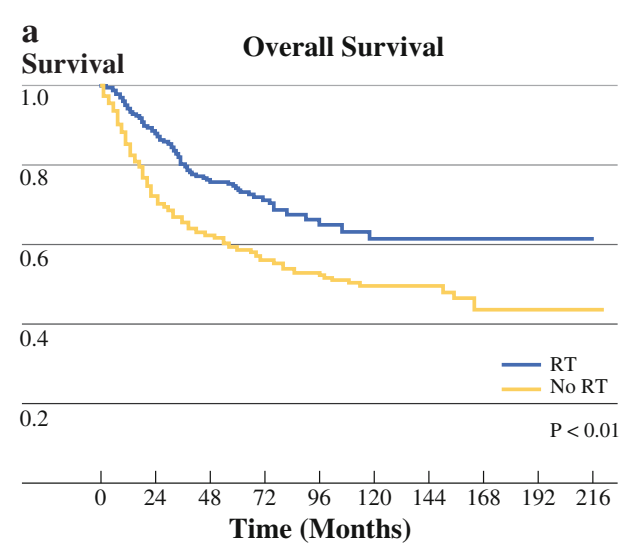

FIG. 2 a Overall survivallumpectomy cohort. b Breastspecific survival—lumpectomy cohort
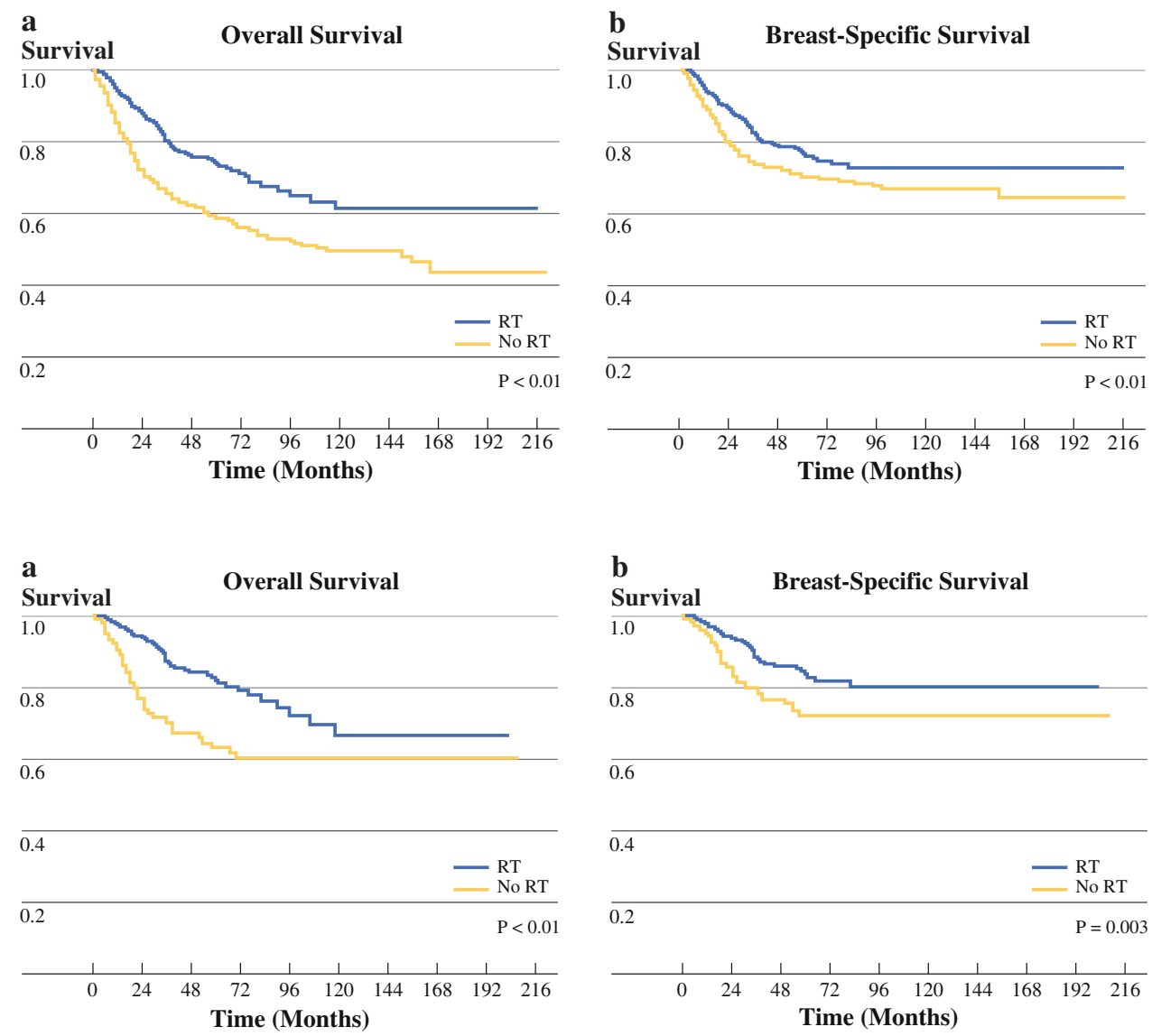

FIG. 3 a Overall survivalmastectomy cohort. b Breastspecific survival—mastectomy cohort
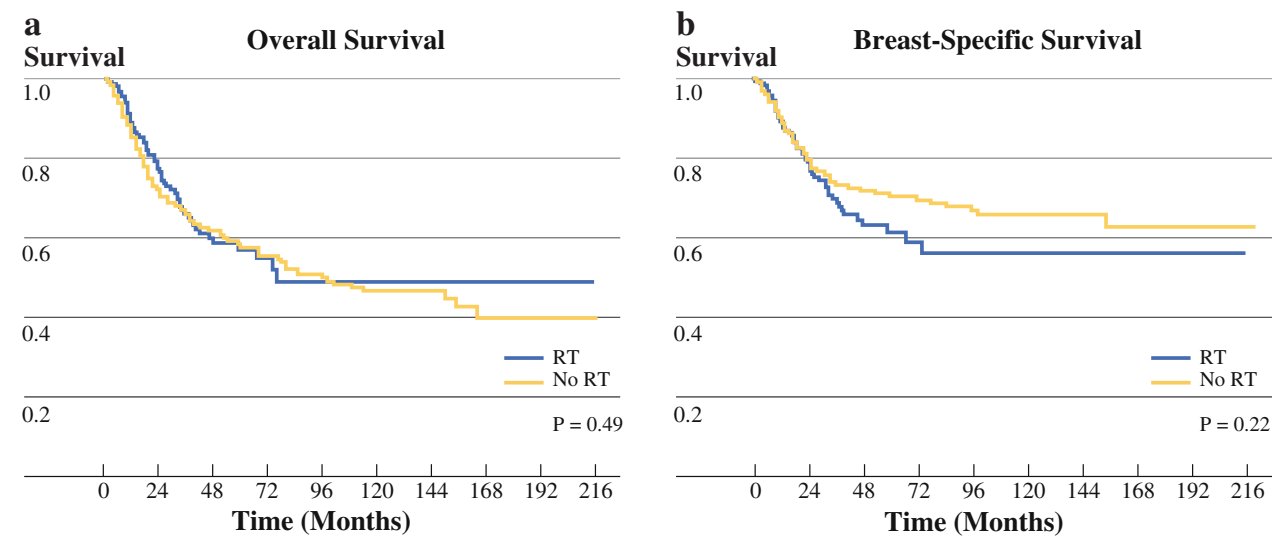

$0.79 ; P<0.01$ ) but not a DSS benefit (HR 0.93, 95\% CI $0.56-1.56 ; P=0.93)$. Likewise, in the mastectomy cohort, an OS benefit (HR 0.67, 95\% CI 0.49-0.90; $P<0.01$ ) but not a DSS benefit was associated with RT (HR 0.73, 95\% CI $0.52-1.02 ; P=0.06)$.

Next, we evaluated whether or not there was a particular group of patients undergoing mastectomy who selectively garnered benefit from RT. Patients receiving mastectomy were separated into two cohorts: "high risk" patients with tumors larger than $5 \mathrm{~cm}$ in size or more than 4 metastatic axillary lymph nodes $(n=338)$ and "normal risk" patients with tumors smaller than $5 \mathrm{~cm}$ in size and fewer than 4 metastatic axillary lymph nodes $(n=438)$. Radiation therapy was received by $39.3 \%$ ( $n=133)$ of "high risk" patients and $12.3 \%(n=54)$ of "normal risk" patients. Multivariate analysis for "high risk" patients revealed RT to be associated with both improved OS (HR 0.53, 95\% CI $0.36-0.77 ; P<0.001)$ and DSS (HR 0.58, 95\% CI 0.38 $0.88 ; P=0.01)$. Multivariate analysis for "normal risk" patients, however, revealed no association between RT and improved OS (HR 1.07, 95\% CI 0.56-2.03; $P=0.83$ ) or DSS (HR 1.46, 95\% CI 0.70-3.02; $P=0.31$ ). 
TABLE 2 Multivariate analysis

\begin{tabular}{|c|c|c|c|c|}
\hline Variable & OS HR & $95 \% \mathrm{CI} / P$ value & DSS HR & $95 \% \mathrm{CI} / P$ value \\
\hline \multicolumn{5}{|l|}{ A: Entire cohort; $n=1501$} \\
\hline Age & 1.02 & $1.02-1.03 ; P<.01$ & 1.01 & $1.00-1.02 ; P<.01$ \\
\hline \multicolumn{5}{|l|}{ Ethnicity/race } \\
\hline Caucasian & 1.00 (referent) & & 1.00 (referent) & \\
\hline Black & 1.00 & $0.75-1.32 ; P=.99$ & 1.00 & $0.73-1.38 ; P=.99$ \\
\hline Asian/Pacific & 0.72 & $0.45-1.13 ; P=.15$ & 0.82 & $0.49-1.37 ; P=.44$ \\
\hline \multicolumn{5}{|l|}{ Islander } \\
\hline Hispanic & 1.45 & $0.96-2.21 ; P=.08$ & 1.47 & $0.91-2.37 ; P=.88$ \\
\hline \multicolumn{5}{|l|}{ Histologic subtype } \\
\hline Metaplastic NOS & 1.00 (referent) & & 1.00 (referent) & \\
\hline Adenocartilaginous & 1.49 & $0.87-2.55 ; P=.15$ & 1.47 & $0.76-2.83 ; P=.25$ \\
\hline Adenospindle & 1.42 & $0.99-2.04 ; P=.06$ & 1.24 & $0.77-1.99 ; P=.37$ \\
\hline Adenosquamous & 1.22 & $0.94-1.59 ; P=.13$ & 1.27 & $0.94-1.74 ; P=.12$ \\
\hline Carcinosarcoma & 1.52 & $1.13-2.04 ; P=.005$ & 1.63 & $1.16-2.31 ; P=.005$ \\
\hline Epithelial & 1.00 & $0.50-2.00 ; P=.99$ & 1.00 & $0.40-2.49 ; P=.99$ \\
\hline Myoepithelial & 1.31 & $0.68-2.54 ; P=.42$ & 1.33 & $0.53-3.34 ; P=.54$ \\
\hline \multicolumn{5}{|l|}{$\mathrm{T}$ stage } \\
\hline $\mathrm{T} 2$ & 1.00 (referent) & & 1.00 (referent) & \\
\hline $\mathrm{T} 1$ & 0.68 & $0.50-0.93 ; P=.02$ & 0.59 & $0.39-0.88 ; P<.01$ \\
\hline $\mathrm{T} 3$ & 2.82 & $2.12-3.75 ; P<.01$ & 2.73 & $1.94-3.86 ; P<.01$ \\
\hline $\mathrm{T} 4$ & 1.95 & $1.45-2.61 ; P<.01$ & 2.28 & $1.62-3.21 ; P<.01$ \\
\hline \multicolumn{5}{|l|}{$\mathrm{N}$ stage } \\
\hline N0 & 1.00 (referent) & & 1.00 (referent) & \\
\hline N1 & 2.40 & $1.83-3.15 ; P<.01$ & 2.71 & $1.98-3.72 ; P<.01$ \\
\hline $\mathrm{N} 2$ & 2.71 & $1.83-4.02 ; P<.01$ & 3.76 & $2.45-5.77 ; P<.01$ \\
\hline N3 & 1.92 & $1.11-3.33 ; P=.02$ & 2.40 & $1.33-4.34 ; P<.01$ \\
\hline Unknown & 2.32 & $1.76-3.06 ; P<.01$ & 2.24 & $1.58-3.20 ; P<.01$ \\
\hline \multicolumn{5}{|l|}{ M stage } \\
\hline M0 & 1.00 (referent) & & 1.00 (referent) & \\
\hline M1 & 4.73 & $3.51-6.39 ; P<.01$ & 5.32 & $3.80-7.45 ; P<.01$ \\
\hline \multicolumn{5}{|l|}{ Tumor grade } \\
\hline III/IV & 1.00 (referent) & & 1.00 (referent) & \\
\hline $\mathrm{I} / \mathrm{II}$ & 0.72 & $0.48-1.06 ; P=.10$ & 0.51 & $0.29-0.89 ; P=.02$ \\
\hline Hormonal receptor status & None significant & & None significant & \\
\hline \multicolumn{5}{|l|}{ Surgery type } \\
\hline Mastectomy & 1.00 (referent) & & 1.00 (referent) & \\
\hline Lumpectomy & 1.02 & $0.78-1.33 ; P=.91$ & 1.20 & $0.87-1.64 ; P=.26$ \\
\hline None & 1.03 & $0.63-1.71 ; P=.90$ & 1.19 & $0.66-2.16 ; P=.56$ \\
\hline \multicolumn{5}{|l|}{ Radiation therapy } \\
\hline No & 1.00 (referent) & & 1.00 (referent) & \\
\hline Yes & 0.64 & $0.51-0.82 ; P<.01$ & 0.74 & $0.56-0.96 ; P<.03$ \\
\hline \multicolumn{5}{|c|}{ B: lumpectomy only; $n=615$} \\
\hline Age & 1.03 & $1.01-1.05 ; \mathrm{p}<0.01$ & 1.00 & $0.98-1.02 ; \mathrm{p}=0.89$ \\
\hline \multicolumn{5}{|l|}{ Ethnicity/race } \\
\hline Caucasian & 1.00 (referent) & & 1.00 (referent) & \\
\hline Black & 1.32 & $0.66-2.64 ; P=.44$ & 1.03 & $0.50-2.14 ; P=.93$ \\
\hline Asian/Pacific & 1.06 & $0.31-3.63 ; P=.93$ & 1.05 & $0.36-3.04 ; P=.93$ \\
\hline
\end{tabular}


TABLE 2 continued

\begin{tabular}{|c|c|c|c|c|}
\hline Variable & OS HR & $95 \% \mathrm{CI} / P$ value & DSS HR & $95 \% \mathrm{CI} / P$ value \\
\hline \multicolumn{5}{|l|}{ Islander } \\
\hline Hispanic & 2.76 & $1.20-6.37 ; P=.02$ & 1.08 & $0.38-3.04 ; P=.89$ \\
\hline \multicolumn{5}{|l|}{ Histologic subtype } \\
\hline Metaplastic NOS & 1.00 (referent) & & 1.00 (referent) & \\
\hline Adenocartilaginous & 1.36 & $0.52-3.56 ; P=.53$ & 1.02 & $0.38-2.75 ; P=.97$ \\
\hline Adenospindle & 1.07 & $0.55-2.11 ; P=.84$ & 1.01 & $0.40-2.58 ; P=.98$ \\
\hline Adenosquamous & 1.85 & $1.03-3.31 ; P=.04$ & 1.07 & $0.58-1.97 ; P=.83$ \\
\hline Carcinosarcoma & 1.46 & $0.14-2.90 ; P=.28$ & 1.09 & $0.50-2.40 ; P=.82$ \\
\hline Epithelial & 0.52 & $0.14-1.91 ; P=.33$ & 0.87 & $0.16-4.59 ; P=.87$ \\
\hline Myoepithelial & 2.25 & $0.80-6.31 ; P=.12$ & 1.02 & $0.24-4.29 ; P=.98$ \\
\hline \multicolumn{5}{|l|}{ T stage } \\
\hline $\mathrm{T} 2$ & 1.00 (referent) & & 1.00 (referent) & \\
\hline $\mathrm{T} 1$ & 0.64 & $0.39-1.04 ; P=.07$ & 0.97 & $0.59-1.58 ; P=.89$ \\
\hline $\mathrm{T} 3$ & 3.59 & $1.12-11.57 ; P=.03$ & 1.13 & $0.22-5.92 ; P=.88$ \\
\hline $\mathrm{T} 4$ & 1.37 & $0.66-2.88 ; P=.40$ & 0.98 & $0.27-3.54 ; P=.98$ \\
\hline \multicolumn{5}{|l|}{$\mathrm{N}$ stage } \\
\hline No & 1.00 (referent) & & 1.00 (referent) & \\
\hline N1 & 2.61 & $1.29-5.25 ; P<.01$ & 1.17 & $0.55-2.50 ; P=.68$ \\
\hline N2 & 11.68 & $4.74-28.73 ; P<.01$ & 1.75 & $0.37-8.35 ; P=.49$ \\
\hline $\mathrm{N} 3$ & 18.18 & $6.74-49.06 ; P<.01$ & 2.10 & $0.29-15.42 ; P=.47$ \\
\hline Unknown & 2.86 & $1.68-4.89 ; P<.01$ & 1.12 & $0.56-2.24 ; P=.76$ \\
\hline \multicolumn{5}{|l|}{ M stage } \\
\hline M0 & 1.00 (referent) & & 1.00 (referent) & \\
\hline M1 & 39.84 & $22.05-72.00 ; P<.01$ & 6.27 & $1.87-21.06 ; P<.01$ \\
\hline \multicolumn{5}{|l|}{ Tumor grade } \\
\hline III/IV & 1.00 (referent) & & 1.00 (referent) & \\
\hline $\mathrm{I} / \mathrm{II}$ & 0.56 & $0.27-1.15 ; P=.12$ & 0.91 & $0.44-1.88 ; P=.79$ \\
\hline Hormonal receptor status & None significant & & None significant & \\
\hline \multicolumn{5}{|l|}{ Radiation therapy } \\
\hline No & 1.00 (referent) & & 1.00 (referent) & \\
\hline Yes & 0.51 & $0.32-0.79 ; P<.01$ & 0.93 & $0.56-1.56 ; P=.79$ \\
\hline \multicolumn{5}{|c|}{ C: mastectomy only; $n=833$} \\
\hline Age & 1.02 & $1.01-1.03 ; P<.01$ & 1.01 & $0.99-1.01 ; P=.14$ \\
\hline \multicolumn{5}{|l|}{ Ethnicity/race } \\
\hline Caucasian & 1.00 (referent) & & 1.00 (referent) & \\
\hline Black & 1.04 & $0.75-1.45 ; P=.81$ & 1.10 & $0.74-1.60 ; P=.66$ \\
\hline Asian/Pacific & 0.78 & $0.47-1.32 ; P=.36$ & 0.86 & $0.46-1.59 ; P=.63$ \\
\hline \multicolumn{5}{|l|}{ Islander } \\
\hline Hispanic & 1.33 & $0.55-3.09 ; P=.39$ & 1.50 & $0.79-2.69 ; P=.23$ \\
\hline \multicolumn{5}{|l|}{ Histologic subtype } \\
\hline Metaplastic NOS & 1.00 (referent) & & 1.00 (referent) & \\
\hline Adenocartilaginous & 1.75 & $0.86-3.57 ; P=.12$ & 1.75 & $0.69-4.45 ; P=.24$ \\
\hline Adenospindle & 1.56 & $0.99-2.46 ; P=.06$ & 1.18 & $0.64-2.17 ; P=.59$ \\
\hline Adenosquamous & 1.00 & $0.71-1.40 ; P=.99$ & 1.02 & $0.69-1.52 ; P=.91$ \\
\hline Carcinosarcoma & 1.35 & $0.95-1.91 ; P=.09$ & 1.45 & $0.96-2.21 ; P=.08$ \\
\hline Epithelial & 0.95 & $0.37-2.42 ; P=.91$ & 1.32 & $0.47-3.76 ; P=.60$ \\
\hline Myoepithelial & 0.68 & $0.21-2.21 ; P=.52$ & 1.00 & $0.23-4.30 ; P=.99$ \\
\hline
\end{tabular}


TABLE 2 continued

\begin{tabular}{|c|c|c|c|c|}
\hline Variable & OS HR & $95 \% \mathrm{CI} / P$ value & DSS HR & $95 \% \mathrm{CI} / P$ value \\
\hline \multicolumn{5}{|l|}{ T stage } \\
\hline $\mathrm{T} 2$ & 1.00 (referent) & & 1.00 (referent) & \\
\hline $\mathrm{T} 1$ & 0.89 & $0.56-1.39 ; P=.60$ & 0.56 & $0.29-1.11 ; P=.10$ \\
\hline $\mathrm{T} 3$ & 2.96 & $2.13-4.11 ; P<.01$ & 2.95 & $1.97-4.40 ; P<.01$ \\
\hline $\mathrm{T} 4$ & 2.19 & $1.55-3.08 ; P<.01$ & 2.74 & $1.82-4.12 ; P<.01$ \\
\hline \multicolumn{5}{|l|}{$\mathrm{N}$ stage } \\
\hline N0 & 1.00 (referent) & & 1.00 (referent) & \\
\hline N1 & 2.28 & $1.65-3.14 ; P<.01$ & 2.41 & $1.65-3.52 ; P<.01$ \\
\hline $\mathrm{N} 2$ & 2.44 & $1.56-3.81 ; P<.01$ & 3.14 & $1.92-5.15 ; P<.01$ \\
\hline N3 & 1.70 & $0.89-3.26 ; P=.11$ & 1.87 & $0.92-3.79 ; P=.08$ \\
\hline Unknown & 2.47 & $1.72-3.55 ; P<.01$ & 12.12 & $1.31-3.45 ; P<.01$ \\
\hline \multicolumn{5}{|l|}{ M stage } \\
\hline M0 & 1.00 (referent) & & 1.00 (referent) & \\
\hline M1 & 4.15 & $2.89-5.97 ; P<.01$ & 4.25 & $2.82-6.40 ; P<.01$ \\
\hline \multicolumn{5}{|l|}{ Tumor grade } \\
\hline III/IV & 1.00 (referent) & & 1.00 (referent) & \\
\hline $\mathrm{I} / \mathrm{II}$ & 0.82 & $0.49-1.36 ; P=.43$ & 0.78 & $0.41-1.50 ; P=.46$ \\
\hline Hormonal receptor status & None significant & & None significant & \\
\hline \multicolumn{5}{|l|}{ Radiation therapy } \\
\hline No & 1.00 (referent) & & 1.00 (referent) & \\
\hline Yes & 0.67 & $0.49-0.90 ; P<.01$ & 0.73 & $0.52-1.02 ; P=.06$ \\
\hline
\end{tabular}

TABLE 3 Effect of radiation therapy on multivariate analysis among several surgical cohorts

\begin{tabular}{lllll}
\hline Cohort & OS HR & $95 \% \mathrm{CI} / P$ value & DSS HR & $95 \% \mathrm{CI} / P$ value \\
\hline Entire cohort & 0.64 & $0.51-0.82 ; P<.01$ & 0.74 & $0.56-0.96 ; P<.03$ \\
Lumpectomy only & 0.51 & $0.32-0.79 ; P<.01$ & 0.93 & $0.56-1.56 ; P=.79$ \\
Mastectomy only & 0.67 & $0.49-0.90 ; P<.01$ & 0.73 & $0.52-1.02 ; P=.06$ \\
\hline
\end{tabular}

Multivariate analyses were repeated for each cohort excluding patients with M1 disease $(6.1 \%, n=92)$. Overall, for the entire cohort, the association between RT and improved survival persisted for both OS (HR 0.62; $P \leq 0.001,95 \%$ CI $0.48-0.81$ ) and DSS (HR 0.66; $P \leq 0.01,95 \%$ CI $0.49-0.90$ ).

Era of treatment was not a significant factor predicting OS or DSS (HR 1.16, 95\% CI $0.85-1.59 ; P=0.34$ and HR $1.14,95 \%$ CI $0.79-1.65 ; P=0.48)$.

\section{DISCUSSION}

Other than a lower rate of lymph node metastases, MBC tumors display poorer prognostic features relative to IDC. $8,11,14,15$ This more aggressive prognostic profile prompted us to examine if the use of adjuvant RT would lead to improvements in OS and DSS.

Results of our multivariate analysis of the entire patient cohort indicated that RT improved both OS and DSS.
Patients receiving RT demonstrated 36 and $26 \%$ decreases in death from any cause and breast-related mortality, respectively. Results of multivariate analyses excluding patients with metastatic disease paralleled these findings, demonstrating 38 and 34\% decreases in death from any cause and breast-related mortality, respectively.

We recognize heterogeneity in our study population. Just over $40 \%$ of patients received lumpectomy for primary surgical treatment, while more than $55 \%$ received mastectomy. To ensure that the survival benefit attributed to the use of RT was not due to the differential use of RT receiving one type of surgery over another, we repeated our multivariate analyses after stratifying patients according to their surgical procedure. Indications for the use of RT are different for patients receiving lumpectomy compared with mastectomy.

Postlumpectomy RT is a standard component of breast conservation therapy for treating IDC to minimize local recurrence. ${ }^{16}$ In our study, approximately $62 \%$ of lumpectomy patients received RT, suggesting the need for 
further education for both physicians and patients. Unlike the NSABP B-06 trial, which did not find a survival advantage for patients with IDC receiving postlumpectomy RT, our study demonstrated that lumpectomy patients receiving RT had a $49 \%$ decreased risk of death from any cause mortality, reflecting the biological differences between MBC and IDC. MBC may be in the spectrum of basal-like breast cancers, which are commonly of higher grade and display more rapid growth. ${ }^{17-20} \mathrm{MBC}$ cells express lower levels of estrogen and progesterone receptors, HER-2/neu receptors and express higher levels of $\mathrm{Ki}$ 67 and p-53 compared to IDC cell lines. ${ }^{9,21}$ Genomic profiling has shown downregulation of DNA repair pathways including BRCA1 pathway, PTEN, and TOP2A. ${ }^{18,22}$ These findings may explain MBC's lower incidence of lymphatic spread, resistance to traditional chemotherapy regimens, and sensitivity to external beam RT.

Postmastectomy RT is recommended to patients with 4 or more metastatic axillary nodes, gross extracapsular nodal extension, large $(\geq 5 \mathrm{~cm})$ primary tumors, and chest wall invasion. ${ }^{23-25}$ In our study, roughly $25 \%$ of mastectomy patients received RT. Mastectomy patients who received RT demonstrated a 33\% decreased risk of death from all-cause mortality. Subgroup analysis of "high" and "normal" risk patients showed that those undergoing mastectomy with tumors $\geq 5 \mathrm{~cm}$ or 4 or more metastatic axillary lymph nodes derived a 47 and $42 \%$ decreased risk of death from any cause and breast-related mortality, respectively. Patients undergoing mastectomy with tumors $\leq 5 \mathrm{~cm}$ and fewer than 4 metastatic axillary lymph nodes, however, derived no benefit from RT. Our data suggest that RT should be considered as a component of multimodality therapy for MBC patients with these advanced features undergoing mastectomy.

Pezzi et al. examined characteristics of patient presenting with MBC using the National Cancer Database and concluded that, compared with patients with IDC, patients with $\mathrm{MBC}$ presented with larger tumor size, less nodal involvement, and less hormonal receptor postivity. ${ }^{8}$ Our data largely support these findings. Our patients presented with large tumors; the median size was $3 \mathrm{~cm}$ and more than $67 \%$ were T stage T2 or greater. Approximately $62 \%$ had N0 disease, and approximately $70 \%$ of tumors were ER/PR negative. Pezzi et al. reported N0 status for $78 \%$ of their patients and negative hormone receptor status in 89-90\% of their patients. Discrepancies with our data are possibly due to the fact that $15 \%$ of our patients had unknown nodal status and approximately $18 \%$ had unknown hormone receptor status. Our study was more inclusive than that of Pezzi et al., who only analyzed tumors coded with ICD-0-3 codes $8575 / 3$. Our study included tumors with more heterogeneous histologic diagnoses. In addition to the ICD-03 code for metaplastic breast cancer, we included codes representing the following histologic subtypes: metaplastic carcinoma not otherwise specified, carcinosarcoma, malignant myoepithelioma, adenosquamous carcinoma, epithelial-myoepithelial, and adenocarcinoma with squamous/cartilaginous and spindle cell metaplasia. These are more in line with the histologic distinctions described by Wargotz et al., who subcategorized MBC into 5 types: matrix producing, spindle like, squamous with ductal origin, metaplastic with osteoclastic giant cells, and carcinosarcoma. $^{2-6}$ In our analysis, carcinosarcoma was associated with both poorer OS (HR 1.52, 95\% CI 1.13-2.04; $P=$ 0.005 ) and DSS (HR 1.63, 95\% CI 1.16-2.31; $P=0.005)$ in the multivariate analysis among all patients (Table 2A). These poorer survivals, however, were not seen upon subgroup analysis, although there was a trend toward significance within the mastectomy-only multivariate analysis for poorer OS (HR 1.35, 95\% CI 0.95-1.91; $P=0.09$ ) and DSS (HR 1.45, 95\% CI 0.96-2.21; $P=0.08$ ). These findings may reflect biological differences between the nonepithelial sarcomatous element found in carcinosarcoma compared with the nonepithelial components of the other histological variants. It is possible that the number of cases of carcinosarcoma is underestimated in our study. It is plausible that the metaplastic NOS histologic subtype represents an admixture of carcinosarcoma and other lower-grade histologies. This heterogeneity of histologic types within the metaplastic NOS category may be responsible for the observed absence of impact on survival reported for metaplastic NOS.

The entire cohort of patients was divided into 2 eras: era I patients representing those diagnosed in 2000 or earlier and era II representing those treated after 2000. No differences in outcomes were found between the 2 groups, suggesting that MBC and combined epithelial-mesenchymal histologic diagnoses from before and after 2000 are similar entities.

Our results should be interpreted with an understanding of its limitations. We excluded cases of MBC prior to 1988 because prior to 1988 , data fields were less complete. As is, data completeness could have influenced our data. Most notably, T stage was unknown for $3.9 \%$, N stage for $15.3 \%$, tumor grade for $20.2 \%$, estrogen receptor status for $17.8 \%$, and progesterone receptor status for $18.5 \%$. Of these, only $\mathrm{T}$ stage and $\mathrm{N}$ stage could potentially influence the decision to use adjuvant RT, making the significance of this missing data less likely. Furthermore, while we have good information regarding the type of treatment patients received, we do not have data on the appropriateness or adequacy of that treatment for individual patients. ${ }^{26}$ Our current analyses could not completely account for all biases that could potentially select for healthier patients in the cohort receiving RT. Furthermore, histopathology data from SEER is not centrally reviewed. Histologic subtypes 
studied in the current analysis represent those reported by the examining pathologists. We contend that our report reflects real-world outcomes. Unlike single-institutionbased studies that are subject to referral bias, our study represents what is occurring at all levels of healthcare institutions and may be more generalizable. Additionally, we do not know whether or not patients received adjuvant medical therapy. Because of the high incidence of hormone receptor negativity in MBC, hormonal therapy is unlikely to influence survival. Previous studies have shown that 33$86 \%$ of MBC patients receive chemotherapy and are twice as likely to receive chemotherapy as matched controls with IDC. ${ }^{8,11,27}$ These tumors are largely chemoresistant, however. $^{27}$ A Mayo clinic report of 27 patients treated over 20 years showed that $33 \%$ received chemotherapy that resulted in one partial response. ${ }^{11}$

Our data suggest that RT should be included in the multimodality treatment for $\mathrm{MBC}$ patients undergoing breast conservation surgery and those patients with tumors $\geq 5 \mathrm{~cm}$ or greater than 4 metastatic axillary lymph nodes undergoing mastectomy. Prospective studies, though needed, are unlikely to be done due to the rarity of MBC.

ACKNOWLEDGMENT Supported by the Landgraf Memorial Research Award and Grant Number UL1RR024146 from the National Center for Research Resources (NCRR) a component of the National Institutes of Health (NIH), and NIH Roadmap for Medical Research. The content is solely the responsibility of the authors and does not necessarily represent the official views of the NNCRR or NIH. Information on NCRR is available at http://www.ncrr.nih.gov/. Information on Re-engineering the Clinical Research Enterprise can be obtained from http://nihroadmap.nih.gov/clinicalresearch/overview-translational.asp.

OPEN ACCESS This article is distributed under the terms of the Creative Commons Attribution Noncommercial License which permits any noncommercial use, distribution, and reproduction in any medium, provided the original author(s) and source are credited.

\section{REFERENCES}

1. Oberman HA. Metaplastic carcinoma of the breast. A clinicopathologic study of 29 patients. Am J Surg Pathol. 1987;11: 918-29.

2. Wargotz ES, Norris HJ. Metaplastic carcinomas of the breast. I. Matrix-producing carcinoma. Hum Pathol. 1989;20:628-35.

3. Wargotz ES, Deos PH, Norris HJ. Metaplastic carcinomas of the breast. II. Spindle cell carcinoma. Hum Pathol. 1989;20:732-40.

4. Wargotz ES, Norris HJ. Metaplastic carcinomas of the breast. III. Carcinosarcoma. Cancer. 1989;64:1490-9.

5. Wargotz ES, Norris HJ. Metaplastic carcinomas of the breast. IV. Squamous cell carcinoma of ductal origin. Cancer. 1990;65: $272-6$.

6. Wargotz ES, Norris HJ. Metaplastic carcinomas of the breast: V. Metaplastic carcinoma with osteoclastic giant cells. Hum Pathol. 1990;21:1142-50.

7. Tavassoli FA. Classification of metaplastic carcinomas of the breast. Pathol Annu. 1992;27:89-119.
8. Pezzi CM, Patel-Parekh L, Cole K, Franko J, Klimberg VS, Bland K. Characteristics and treatment of metaplastic breast cancer: analysis of 892 cases from the National Cancer Data Base. Ann Surg Oncol. 2007;14:166-73.

9. Barnes PJ, Boutilier R, Chiasson D, Rayson D. Metaplastic breast carcinoma: clinical-pathologic characteristics and HER2/neu expression. Breast Cancer Res Treat. 2005;91:173-8.

10. Luini A, Aguilar M, Gatti G, Fasani R, Botteri E, Brito JA, et al. Metaplastic carcinoma of the breast, an unusual disease with worse prognosis: the experience of the European Institute of Oncology and review of the literature. Breast Cancer Res Treat. 2007;101:349-53.

11. Rayson D, Adjei AA, Suman VJ, Wold LE, Ingle JN. Metaplastic breast cancer: prognosis and response to systemic therapy. Ann Oncol. 1999;10:413-9.

12. Dave G, Cosmatos H, Do T, Lodin K, Varshney D. Metaplastic carcinoma of the breast: a retrospective review. Int $J$ Radiat Oncol Biol Phys. 2006;64:771-5.

13. Gutman H, Pollock RE, Janjan NA, Johnston DA. Biologic distinctions and therapeutic implications of sarcomatoid metaplasia of epithelial carcinoma of the breast. J Am Coll Surg. 1995;180: 193-9.

14. Wargotz ES, Norris HJ. Metaplastic carcinomas and sarcomas of the breast. Am J Clin Pathol. 1991;96:781.

15. Gibson GR, Qian D, Ku JK, Lai LL. Metaplastic breast cancer: clinical features and outcomes. Am Surg. 2005;71:725-30.

16. Fisher B, Anderson S, Bryant J, Margolese RG, Deutsch M, Fisher ER, et al. Twenty-year follow-up of a randomized trial comparing total mastectomy, lumpectomy, and lumpectomy plus irradiation for the treatment of invasive breast cancer. $N$ Engl $J$ Med. 2002;347:1233-41.

17. Kuroda N, Fujishima N, Inoue K, Ohara M, Hirouchi T, Mizuno $\mathrm{K}$, et al. Basal-like carcinoma of the breast: further evidence of the possibility that most metaplastic carcinomas may be actually basal-like carcinomas. Med Mol Morphol. 2008;41:117-20.

18. Weigelt B, Kreike B, Reis-Filho JS. Metaplastic breast carcinomas are basal-like breast cancers: a genomic profiling analysis. Breast Cancer Res Treat. 2009;117:273-80.

19. Reis-Filho JS, Milanezi F, Steele D, Savage K, Simpson PT, Nesland JM, et al. Metaplastic breast carcinomas are basal-like tumours. Histopathology. 2006;49:10-21.

20. Korsching E, Jeffrey SS, Meinerz W, Decker T, Boecker W, Buerger H. Basal carcinoma of the breast revisited: an old entity with new interpretations. J Clin Pathol. 2008;61:553-60.

21. Tse GM, Tan PH, Putti TC, Lui PC, Chaiwun B, Law BK. Metaplastic carcinoma of the breast: a clinicopathological review. J Clin Pathol. 2006;59:1079-83.

22. Rouzier R, Perou CM, Symmans WF, Ibrahim N. Cristofanilli M, Anderson $\mathrm{K}$, et al. Breast cancer molecular subtypes respond differently to preoperative chemotherapy. Clin Cancer Res. 2005;11:5678-85.

23. Ragaz J, Jackson SM, Le N, Plenderleith IH, Spinelli JJ, Basco $\mathrm{VE}$, et al. Adjuvant radiotherapy and chemotherapy in nodepositive premenopausal women with breast cancer. $N$ Engl J Med. 1997;337:956-62.

24. Overgaard M, Hansen PS, Overgaard J, Rose C, Andersson M, Bach $\mathrm{F}$, et al. Postoperative radiotherapy in high-risk premenopausal women with breast cancer who receive adjuvant chemotherapy. Danish Breast Cancer Cooperative Group 82b Trial. $N$ Engl J Med. 1997;337:949-55.

25. Fowble B, Gray R, Gilchrist K, Goodman RL, Taylor S, Tormey DC. Identification of a subgroup of patients with breast cancer and histologically positive axillary nodes receiving adjuvant chemotherapy who may benefit from postoperative radiotherapy. J Clin Oncol. 1988;6:1107-17. 
26. Yu JB, Gross CP, Wilson LD, Smith BD. NCI SEER public-use data: applications and limitations in oncology research. Oncology (Williston Park). 2009;23:288-95.
27. Hennessy BT, Giordano S, Broglio K, Duan Z, Trent J, Buchholz TA, et al. Biphasic metaplastic sarcomatoid carcinoma of the breast. Ann Oncol. 2006;17:605-13. 